\title{
Echelons of Some Haemoparasites among Blood Donors in Port Harcourt, Rivers State, Nigeria
}

\author{
Evelyn Mgbeoma Eze*, Serekara Gideon Christian, Ransom Baribefii Jacob, Barinaaziga \\ Sunday Mbeera, Beatrice Wobiarueri Moore-Igwe and Angela Tornubari Mbari
}

Department of Medical Laboratory Science, Rivers State University, Port-Harcourt, Nigeria

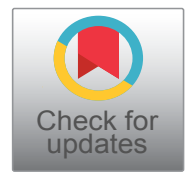

*Corresponding author: Evelyn Mgbeoma Eze, Department of Medical Laboratory Science, Rivers State University, PortHarcourt, Nigeria, Tel: +2347066754280

\begin{abstract}
Although the therapeutic application of whole blood and blood components can be life-saving, inadequate screening of these products could pose life-threatening problems to the recipient. The aim of this study was to determine the echelons of some haemoparasites (malaria parasite, microfilaria and babesia species) among blood donors in Port Harcourt, Rivers State, Nigeria as well as quantifying their densities. A total of one hundred (100) prospective blood donors from the participating blood banks within 1951 years were recruited for this study. Two milliliters $(2 \mathrm{mls})$ of venous blood was collected from the antecubital vein of each participant using standard venepuncture technique into ethylene diamine tetra acetic acid (EDTA) bottles and mixed properly to avoid blood clotting. Thick and thin blood films were used for the detection of haemoparasites. The data generated was analysed using statistical package for Social Sciences (SPSS) version 20. Out of 100 samples examined, $23(23.0 \%)$ were positive for Plasmodium falciparum. The highest prevalence was among the males $13(13.0 \%)$, between the ages of $19-29$ years and only $10(10.0 \%)$ of the females were positive while the lowest prevalence was between the ages of $41-51$. No positive case was observed for microfilaria and babesia species. The mean malaria parasite density for male subjects was $0.43 \pm 0.23 \%$ while that of female subjects was 0.66 $\pm 0.23 \%$. The female subjects had significantly higher malaria parasite density than the males $(P=0.03)$. The data obtained from this study provides information on the haemoparasite status, indicating level of malaria parasite among the prospective blood donors in Port Harcourt, Nigeria. It is therefore, recommended that malaria parasite screening test be included among other blood screening tests before any transfusion to avert the deleterious effects of malaria parasite on the recipient and to enhance a more safe blood for the purpose of transfusion.
\end{abstract}

\section{Keywords}

Haemoparasites, Echelons, Malaria, Microfilaria, Babesia species, Blood, Donors, Parasite density

\section{Introduction}

Transfusion of blood is an essential therapeutic procedure everywhere in the world as it is a life saving measure but in some instances, it transmits parasitic infections that are lethal. The field of transfusion medicine has encountered a huge problem in providing safe blood and blood product and blood banks as well as plasma manufacturing industries have aggressively pursued strategies in order to reduce the risk of transfusing a haemoparasitized blood. There are many haemoparasites but the most common parasitic organisms implicated in transfusion science are Plasmodium falciparum, Wuchereria bancrofti and Babesiamicroti $[1,2]$. In order for these parasites to be transmitted by blood transfusion, they must circulate in the blood stream of the donors, comprise of certain physical characteristics and survive conservation in order to generate infection in the blood.

Malaria is a mosquito-borne infectious disease caused by four Plasmodium species in humans; Plasmodium falciparum, Plasmodium vivax, Plasmodium ovale and Plasmodium malariae through the bite of an infected female Anopheles mosquito while the species Plasmodium knowlesi rarely causes diseases in humans [3]. Most deaths are caused by Plasmodium falciparum

Citation: Eze EM, Christian SG, Jacob RB, Mbeera BS, Moore-Igwe BW, et al. (2021) Echelons of Some Haemoparasites among Blood Donors in Port Harcourt, Rivers State, Nigeria. Int J Blood Res Disord 8:070. doi.org/10.23937/2469-5696/1410070

Accepted: December 02, 2021: Published: December 04, 2021

Copyright: (C) 2021 Eze EM, et al. This is an open-access article distributed under the terms of the Creative Commons Attribution License, which permits unrestricted use, distribution, and reproduction in any medium, provided the original author and source are credited. 
because other species causes a milder form of the disease. Plasmodium falciparum does not have a fixed structure as it changes continuously during its life cycle [4]. It has an incubation period of 11 days but may range from 9-30 days with clinical symptoms such as fever, chills, headache, sweating, dizziness, malaise, nausea, vomiting and mild diarrhea [5]. Plasmodium falciparum is widely distributed in all continents except Europe and diagnosis is based on the microscopic examination of blood utilizing blood film [6]. Combination of drugs such as Artemether/Lumefantrine, Artesunate/Amodiquine can be used as a treatment option [7].

Filariases, another parasitic disease caused by an infection with round worms of filaria type are spread by blood-feeding black flies and mosquitoes. Three parasitic round worms (Wuchereria bancrofti, Brugiamalayi and Brugiatimori) cause lymphatic filariasis and are spread by a variety of mosquito vector species. Wuchereria bancrofti being the most prevalent of these species as lymphatic filariasis affects more than 120 million people worldwide [8]. The parasite exhibits sexual dimorphism of which females are ovoviviparous, producing thousands of juveniles called microfilaria [9]. They carry out their life cycle in two hosts (humans and mosquitoes) and they are sheathed, thus, enabling their identification. They also exhibit a unique diurnal periodicity [10]. The presence of fever, axillary lymphadenopathy, skin exfoliation, limb and genital swelling are the clinical presentation of the disease.

Filaria diagnosis is achieved by identifying microfilaria on a Giemsa stained thin and thick blood film [11] and treated by administration of albendazole in combination with ivermectin [12].

Lastly, Babesiosis is a malaria-like parasitic disease caused by infection with Babesia, transmitted by the bite of Ixodes scalpularis ticks [13]. The two species that frequently infect humans are Babesia microtiand Babesia divergens and symptoms include fever, haemolytic anaemia, chills, sweats, thrombocytopenia that may last for several days to months, it is mostly encountered in the United States, Fire Island and Eastern Long Island and usually requiring 2 hosts (a white-footed mouse and a tick) for its life cycle. The sporozoites become introduced into the host (mouse) during a blood meal, undergo morphological changes and multiply, leading to the clinical manifestation of the disease [14]. The identification of this parasite on a Giemsa stained thin blood film is a definitive diagnostic test and treatment involves a combination of atovaquone and azithromycin [15].

Parasite density estimations can be performed using thick or thin films, each method having its limitations. The estimation of parasite density from the thin film tends to be more accurate than those estimated from thick film because there is little or no parasite loss during staining [16]. However, the thin film method is mostly appropriate for higher parasite densities only. Parasite density estimation from thick film may beless accurate due to parasite loss but can be adapted for use with a wide range of parasite densities at the medium to lower end of detectable parasitaemia. Thin films will be more accurate with higher levels of parasitaemia, as it may be difficult to obtain precise parasite counts from thick films with these high parasite densities. In determining parasite density from thick film, the methods using the white cell count, the 'per high power field' (HPF) method or the Earle-Perez method may be used [17]. The Earle-Perez method is a method developed in 1932 for determining parasite density from the thick film, which can be used when actual WBC counts are not available [17].

Warhurst and Williams [18] discussed several methods employed in quantifying parasites in thick blood smears. One technique is to count the total number of parasites per 200 WBC and multiplying it by 40 to give the number of parasites per microlitre assuming that there are $8000 \mathrm{WBC} /$ microlitre blood. A second method involves making a thick smear with a known volume of blood (5 microlitres) and staining it with Giemsa stain before counting all the parasites on the smear. The total parasite counted is divided by 5 to obtain the number of parasites per microlitre. A similar technique uses a defined amount of whole blood to make a thick smear and then the parasites present in $100 \mathrm{HPFs}$ are counted. One parasite per $100 \mathrm{HPF}$ is assumed to be equivalent to 50 parasites per microlitre. A thick smear is generally considered negative if no parasites are seen after 10 minutes searching. Parasite levels may also be quantified by examination of a thin blood smear which is accomplished by counting the number of parasites per 1000 RBCs and expressing as a percentage, the number of erythrocytes infected or by counting the number of parasites per 200 WBCs as described for thick film [18].

Consequently, the screening of blood donors and safety of donated blood component is paramount in diagnostic and therapeutic laboratory medicine. Following records of transmission of blood borne diseases in blood banks, it becomes necessary to synthesize relevant information in other to ascertain the true state of this assertions and thus this research work is aimed at determining the echelons of some haemoparasites (malaria parasite, microfilaria and babesia species) among blood donors in Port Harcourt, Rivers State, Nigeria as well as quantifying their densities.

\section{Materials and Methods}

\section{Study design}

A cross-sectional study design was employed.

\section{Study area}

This research study was carried out in Braithwaite Memorial Specialist Hospital (BMSH) now Rivers State 
University Teaching Hospital (RSUTH), Port Harcourt, Nigeria. Port Harcourt is the current capital and largest city of Rivers State, which lies along the Bonny River and is located in the Niger Delta with a latitude of $4^{\circ} 46^{\prime} 38^{\prime \prime} \mathrm{N}$ and a longitude of $7^{\circ} 00^{\prime} 48^{\prime \prime} E$ and as at 2016, the Port Harcourt urban area had an estimated population of $1,865,000$ inhabitants.

\section{Study population}

A total of 100 apparently healthy prospective blood donors comprising of seventy-seven (77) males and twenty-three (23) females within the ages of 19-52 years were recruited into the study. Verbal and written consent was obtained from donors and the Head of Department respectively. Apparently healthy donors aged 19-52 years who have met all the donor eligibility criteria were included in this study. Prospective donors who were less than 18 years of age or more than 52 years, whose body weight was less than $50 \mathrm{~kg}$, whose haemoglobin level was less than $12.5 \mathrm{~g} / \mathrm{dl}$, those with history of jaundice and sickle cell disease, chain smokers and those with tattoo were excluded from this study.

\section{Sample collection}

Two mililitres ( $2 \mathrm{mls}$ ) of donor's venous blood was collected into an ethylene diamine tetra-acetic acid (EDTA) sample bottle with a concentration of $1.5 \mathrm{mg} /$ $\mathrm{ml}$ using needle and syringe and transported at room temperature $\left(20-25^{\circ} \mathrm{C}\right)$. Samples were processed using standard laboratory procedures.

\section{Laboratory analysis}

Samples were analyzed by making a thick and thin blood film. Thick blood film was used for the identification of the presence of malaria parasite while thin blood film was used for specie identification. Wet slide preparation was used for the identification of microfilaria. Slides were then stained with diluted Giemsa stain.

Identification of malaria parasites: The method employed was as described by Cavalcanti and Santos [19].

Principle of giemsa stain: The cationic or basic dye binds to anionic sites, giving it a blue-grey colour to the nucleic acids while the anionic or acidic dye binds to cationic sites on proteins and gives an orange-red colour to haemoglobin and eosinophil granules.

Procedure for thick and thin film preparation: Two (2) drops of blood was added on a clean dry grease free glass slide. Blood was spread in a circle evenly to obtain a coin-shape. Film was allowed to air dry. Slide was dehaemoglobinized and labelled with a grease free pencil. A drop of blood was placed on one end of a clean dry slide. A clean smooth edged spreader was placed on the slide and drawn backwards to touch the drop of blood. The blood was allowed to extend along the edge of the spreader. Holding the spreader at an angle of about $30-45^{\circ} \mathrm{C}$, blood was spread to make a film about 40-50 $\mathrm{mm}$ in length. Film was air dried by waving slide back and forth.

Staining of thick and thin blood film: The method employed was Giemsa staining technique as described by [19].

A 1:20 dilution of Giemsa stain was made with buffered ${ }_{p} \mathrm{H7}$.2 water. Slides were placed facing upwards in a shallow tray supported by 2 rods with the film facing upwards. Slides were flooded with diluted Giemsa stain for 20 minutes. Slides were washed using distilled water. The back of each slide was wiped and place in a draining rack. Preparations were allowed to air dry vertically.

Wet preparation for microfilaria: The method used was as described by Nutmanand Kumaraswami [20].

Procedure: $0.02 \mathrm{ml}$ of blood was mixed with 2 drops of water on a slide. Preparation was covered with a cover glass. Slide was microscopically examined for motile microfilaria using 10X objective.

\section{Identification of babesia species}

Preparation of thick blood film: Two (2) drops of blood was added on clean dry grease free glass slide. Blood was spread in a circle evenly to obtain a coin-shape. Film was allowed to air dry. Slide was dehaemoglobinized and labelled with a grease free pencil.

Staining of thick blood film: The method employed was Giemsa staining technique is as described by [19].

A 1:20 dilution of Giemsa stain was made with buffered $\mathrm{PH} 7.2$ water. Slides were placed facing upwards in a shallow trey supported by 2 rods with the film facing upwards. Slides were flooded with diluted Giemsa stain for 20 minutes. Slides were washed using distilled water. The back of each slide was wiped and placed in a draining rack. Preparation was allowed to air dry vertically.

\section{Calculation of parasite density}

The formula adopted for calculating parasite density is given by Manas, et al. [16] thus:

Parasitaemia $(\%)=\frac{\text { Number of parasitized red blood cells }}{\text { Tota } \ln \text { umber of red blood cells }} \times 100$

Where total number of red blood cells $=500$

\section{Statistical analysis}

The data obtained was analyzed using statistical package for social science (SPSS) version 22. The presence of haemoparasites was expressed as positive and absence was expressed as negative. Descriptive statistics was employed to obtain the percentages of the distribution of the haemoparasites. Mean of the data obtained from donors were analyzed by using student independent 
Table 1: Demographic details of participants.

\begin{tabular}{|l|l|l|l|}
\hline AGE GROUP & NUMBER TESTED & \multicolumn{2}{|l|}{ MALE } \\
\hline $19-29$ & 54 & 38 & 16 \\
\hline $30-40$ & 27 & 26 & 7 \\
\hline $41-51$ & 19 & 13 & 0 \\
\hline TOTAL & $\mathbf{1 0 0}$ & $\mathbf{7 7}$ & $\mathbf{2 3}$ \\
\hline
\end{tabular}

Table 2: Plasmodium falciparum infection according to age groups.

\begin{tabular}{|l|l|l|l|}
\hline AGE RANGE & NO.EXAMINED & NO. INFECTED & \% INFECTED \\
\hline $19-29$ & 45 & 11 & $47.83 \%$ \\
\hline $30-40$ & 36 & 7 & $30.43 \%$ \\
\hline $41-51$ & 19 & 5 & $21.74 \%$ \\
\hline TOTAL & $\mathbf{1 0 0}$ & $\mathbf{2 3}$ & $\mathbf{1 0 0}$ \\
\hline
\end{tabular}

Table 3: Plasmodium falciparum infection according to gender.

\begin{tabular}{|l|l|l|l|}
\hline AGE RANGE & NO.EXAMINED & NO. INFECTED & \% INFECTED \\
\hline MALE & 72 & 13 & $13.00 \%$ \\
\hline FEMALE & 28 & 10 & $10.00 \%$ \\
\hline TOTAL & $\mathbf{1 0 0}$ & $\mathbf{2 3}$ & $\mathbf{2 3 . 0 0 \%}$ \\
\hline
\end{tabular}

Table 4: Comparison of malaria parasite density according to gender.

\begin{tabular}{|l|l|}
\hline GENDER & MALARIA PARASITE DENSITY (\%) \\
\hline MALE & 0.43 \\
\hline FEMALE & 0.66 \\
\hline P-value & 0.03 \\
\hline
\end{tabular}

Table 5: Microfilaria according to age groups.

\begin{tabular}{|l|l|l|}
\hline AGE RANGE & NO.EXAMINED & NO. INFECTED \\
\hline $19-29$ & 45 & 0 \\
\hline $30-40$ & 36 & 0 \\
\hline $41-51$ & 19 & 0 \\
\hline TOTAL & $\mathbf{1 0 0}$ & $\mathbf{0}$ \\
\hline
\end{tabular}

Table 6: Babesiosis according to age groups.

\begin{tabular}{|l|l|l|}
\hline AGE RANGE & NO.EXAMINED & NO. INFECTED \\
\hline $19-29$ & 45 & 0 \\
\hline $30-40$ & 36 & 0 \\
\hline $41-51$ & 19 & 0 \\
\hline TOTAL & 100 & $\mathbf{0}$ \\
\hline
\end{tabular}

T-test to determine the significant difference between the parasite densities of the malaria infected donors based on gender. The presence of haemoparasites was expressed as positive and absence was expressed as negative. Results were also presented in Tables.

\section{Results}

\section{Demographic details of participants}

Venous blood was obtained and tested for malaria parasite, microfilaria and babesia. A total of 100 subjects aged between 19-51 years were recruited in this study, both male $(n=77)$ and female $(n=23)$. Table 1 shows the demographic details of participants.

\section{Plasmodium falciparum infections according to age groups}

Table 2 shows Plasmodium falciparum among age groups. A total of 23 infected cases were identified. For age groups 19-29 there were 11 positive cases out of 45 subjects examined, 30-40 there were 7 positive cases out of 36 subjects examined and for 41-51 there were 5 positive cases out of 19 subjects examined.

\section{Plasmodium falciparum infections according to gender}

A total of 72 males were examined and $13(13 \%)$ were infected, also, a total of 28 females were examined and $10(10 \%)$ were infected. This shows that Plasmodium falciparum infections had high prevalence in males $(13 \%)$ than females $(10 \%)$ as shown in Table 3.

\section{Comparison of malaria parasite density according to gender}

Table 4 shows that the mean malaria parasite density for male subjects was $0.43 \pm 0.23 \%$ while that of female subjects was $0.66 \pm 0.23 \%$. The female subjects had significantly higher malaria parasite density than the males $(P=0.03)$.

\section{Microfilaria according to age groups}

Table 5 shows microfilaria among age groups with no positive case identified.

\section{Babesiosis according to age groups}

Table 6 shows babesiosis among age groups with no positive case identified. 


\section{Discussion}

Plasmodium falciparum was the only specie of malaria parasite identified in this study because it is the most common of the four human malaria parasites in the clime under study. Out of a total of 100 blood donors screened for haemoparasites, 23 (23\%) haboured the malaria parasite Plasmodium falciparum while $0 \%$ haboured microfilaria and babesiosis. Age related prevalence showed that those within the age group of $19-29$ years were more infected $(47.83 \%)$ while those within the age group of 41-51 were the least infected (21-74\%). Gender related prevalence showed that males were more prevalent than females as they had a prevalence of 13.00 and $10.00 \%$ respectively. The mean malaria parasite density for male subjects was $0.43 \pm$ $0.23 \%$ while that of female subjects was $0.66 \pm 0.23 \%$. The female subjects had significantly higher malaria parasite density than the males $(P=0.03)$.

The malaria prevalence of $23 \%$ among blood donors obtained from this study should be a cause for concern because recipients of these bloods are mostly pregnant women, children under 5 years, accident victims and other immuno-suppressed patients coupled with the fact that ninety-five percent (95\%) of transfusions in Nigeria and the entire sub-Saharan Africa according to Owusu-Ofori, et al. [21] utilizes whole blood rather than components. Again, the result of this study is higher than findings of previous studies in the Niger Delta Area by Erhabor, et al. [22]; Pondei, et al. [23] who reported $10.2 \%$ and $12.5 \%$ respectively. However, it agrees with Oladeinde, et al., [24] who reported $27.5 \%$ and disagrees with Uneke, et al. [25] and Epidi, et al. [26] who reported $40.5 \%$ and $51.5 \%$ respectively.

The prevalence of haemoparasites varies from 0.2 cases per million in non-endemic countries to 50 or more cases per million in endemic areas and the prevalence of haemoparasites among blood donors had been reported to be as high as $55 \%$ in the highly endemic areas in Nigeria according to Owusu-Ofori, et al. [21]. The findings in this present study are similar to that of Pondei, et al., [23]; Abah and Temple [27].

Males were more infected with the malaria parasite Plasmodium falciparum because more males ( $n=77$ ) presented themselves for blood donations than females $(n=23)$ and this corroborates Bani and Giussani [28] observations that gender plays a key role in the motivation to give blood; women being more altruistic and men being more individualistic. Thus, fewer women are regular donors. Younger subjects were more infected than older subjects because the former are more susceptible than the later. However, World Health Organization had observed that younger people in malaria endemic areas are more susceptible to malaria infections than older people [29]. It can also be due to the fact that the younger subjects were more exposed to the parasites.

\section{Conclusion}

The prevalence rate of $23 \%$ for malaria parasite among blood donors recorded in this study is of great concern particularly if such blood is transfused to pregnant women, children and immunocompromised patients. It is recommended that screening for malaria parasite be included in the routine investigation of prospective blood donors in Nigeria because breaking the transmission chain from blood donors to recipients is another step in achieving the desired goal of curbing the scourge of malaria infections and by implication reducing malaria related complications and deaths and also enhance safe blood transfusion.

\section{References}

1. Okpokam DC, Inyang UU, Emeribe AO (2019) Prevalence of haemoparasites in blood donors, calabar, nigeria perspective. Asian Journal of Biological Science 12: 385395.

2. Singh G, Sehgal R (2010) Transfusion-transmitted parasitic infections. Asian J Transfus Sci 4: 73-77.

3. Caraballo H (2014) Emergency department management of mosquito-borne illness: Malaria, dengue, and west nile virus. Emergency Medicine Practice 16: 21-25.

4. Lucius R, Roberts CW, Lucius R, Loos-Frank B, Lane $\mathrm{RP}$, et al. (2017) The biology of parasitaemia. Trends in Parasitology 19: 144-149.

5. Trampuz A, Jereb M, Muzlovic I, Prabhu RM (2003) Clinical review: Severe malaria. Critical Care 7: 315-316.

6. Krafts K, Hempelmann E, Oleksyn B (2011) The color purple from royalty to laboratory, with apologies to Malachowski. Biotechnique and Histochemistry 86: 7-35.

7. World Health Organization Guidelines (2010) Guidelines for the treatment of malaria.

8. Centers for Disease Control and Prevention (2011) Progress toward elimination of lymphatic filariasis. 29: 9899.

9. Ethan BC, Harvey LP (2009) Lymphatic filariasis. Parasitology Today 12: 25-26.

10. Ridley JW (2012) Diagnosis and treatment of plasmodium malaria. Parasitology for medical and clinical laboratory professionals 3: 103-104.

11. Ramaiah KD, Ottesen EA (2014) Progress and impact of 13 years of the global programme to eliminate lymphatic filariasis on reducing the burden of filarial disease. PLoS Neglected Tropical Diseases 8: 3316-3319.

12. Chernin E (1987) The disappearance of bancroftian filariasis from Charleston, South Carolina. Am J Trop Med Hyg 37: 111-114.

13. Hunfeld KP, Hildebrandt A, Gray JS (2008) Babesiosis, recent insights into an ancient disease. Int J Parasitol 38: 1219-1237.

14. Centers for Disease Control and Prevention (2015) Life cycle of Babesia microti.

15. Tanyel E, Guler N, Hokelek M, Ulger F, Sunbul M (2015) A case of severe babesiosis treated successfully with exchange transfusion. International Journal of Infectious Diseases 38: 83-85. 
16. Manas K, Duangjal P, Bhukdee P, Nuool P, Chaowanee $C$ et al. (2015) Effects of malaria parasite density on blood cell parameters. PLoS One 10: 1-11.

17. Laurens MB, Duncan CJ, Epstein JE, Hill AV, Komisar JL, et al. (2012) A consultation on the optimization of controlled human malaria infection by mosquito bite. Vaccine 30 : 301 302.

18. Warhurst DC, Williams JE (1996) Laboratory diagnosis of malaria. Journal of Clinical Pathology 49: 533-538.

19. Cavalcanti AJ, Santos BC (2015) Giemsa staining of malaria blood films. British Medical Journal 32: 625-630.

20. Nutman TB, Kumaraswami V (2018) Serpentine movement of filaria in wet mount preparation. Advanced Cytology and Pathology 3: 10-11.

21. Owusu-Ofori AK, Parry C, Bates I (2010) Transfusiontransmitted malaria in countries where malaria is endemic: A review of the litreature from sub-Saharan Africa. Clinical Infectious Diseases 5: 1192-1198.

22. Erhabor O, Ok O, Awah I, Uko KE, Charles AT (2007) The prevalence of Plasmodia parasitaemia among donors in the Niger delta of Nigeria. Trop Doct 37: 32-34.
23. Pondei K, Ebidor L, Eno N (2012) Prevalence of the malaria parasite in screened blood in a tertiary health centre in the malaria-endemic Niger Delta region of Nigeria. Global Advance Research Journal of Microbiology 1: 188-193.

24. Oladeinde BH, Omoregie R, Osakue EO, Onaiwu TO (2014) Asymptomatic malaria among Blood Donors in Benin City Nigeria. Iran J Parasitol 9: 415-422.

25. Uneke CJ, Ogbu O, Nwojiji V (2006) Potential risk of induced malaria by blood transfusion in south-eastern nigeria. Mcgill journal of Medicine 9: 8-13.

26. Epidi TT, Nwani CD, Ugorji NP (2008) Prevalence of malaria in blood donors in Abakaliki. Scientific Research and Essays 3: 162-164.

27. Abah AE, Temple B (2015) Prevalence of malaria parasite among asymptomatic primary school children in angiama community, bayelsa state, nigeria. Tropical Medicine and Surgery 4: 203-207.

28. Bani M, Giussani B (2010) Gender differences in giving blood: A review of the litreature. Blood Transfusion 8: 278287.

29. World Health Organization (2003) African malaria report. 